\title{
Can we consider discontinuation of hypomethylating agents in patients with myelodysplastic syndrome : a retrospective study from The Korean Society of Hematology AML/MDS Working Party
}

\author{
Da Jung Kim¹, Ho Sup Lee ${ }^{1}$, Joon-Ho Moon ${ }^{2}$, Sang Kyun Sohn ${ }^{2}$, Hyeoung Joon Kim³ \\ June-Won Cheong ${ }^{4}$, Deog-Yeon Jo5, Hawk Kim ${ }^{6}$, Hyewon Lee ${ }^{7}$, Soo-Mee Bang ${ }^{8}$, \\ Won Sik Lee ${ }^{9}$, Yong Park ${ }^{10}$, Mark Hong Lee ${ }^{11}$, Jae Hoon Lee ${ }^{12}$, Sung Hwa Bae ${ }^{13}$, Min \\ Kyoung Kim ${ }^{14}$ and The Korean Society of Hematology AML/MDS Working Party \\ ${ }^{1}$ Department of Internal Medicine, Kosin University College of Medicine, Kosin University Gospel Hospital, Busan, South \\ Korea \\ ${ }^{2}$ Department of Hematology/Oncology, Kyungpook National University Hospital, Daegu, South Korea \\ ${ }^{3}$ Department of Hematology-Oncology, Chonnam National University Hwasun Hospital, Hwasun, Jeollanamdo, South Korea \\ ${ }^{4}$ Department of Internal Medicine, Yonsei University College of Medicine, Seoul, South Korea \\ ${ }^{5}$ Department of Internal Medicine, Chungnam National University Hospital, Chungnam National University, Daejeon, South \\ Korea \\ ${ }^{6}$ Division of Hematology and Cellular Therapy, Ulsan University Hospital, University of Ulsan College of Medicine, Ulsan, \\ South Korea \\ ${ }^{7}$ Department of Hematology-Oncology, Center for Hematologic Malignancy, National Cancer Center, Goyang, South Korea \\ ${ }^{8}$ Department of Internal Medicine, Seoul National University Bundang Hospital, Seoul, South Korea \\ ${ }^{9}$ Department of Internal Medicine, Busan Paik Hospital, Busan, South Korea \\ ${ }^{10}$ Department of Internal Medicine, Korea University College of Medicine, Seoul, South Korea \\ ${ }^{11}$ Division of Hematology-Oncology, Department of Internal Medicine, Konkuk University Medical Center, Konkuk University \\ School of Medicine, Seoul, South Korea \\ ${ }^{12}$ Department of Internal Medicine, Gachon University Gil Medical Center, Incheon, South Korea \\ ${ }^{13}$ Department of Internal Medicine, Daegu Catholic University Medical Center, Daegu, South Korea \\ ${ }^{14}$ Department of Hematology-Oncology, Yeungnam University Medical Center, Yeungnam University School of Medicine, \\ Daegu, South Korea \\ Correspondence to: Ho Sup Lee, email: hs52silver@gmail.com \\ Keywords: myelodysplastic syndrome, discontinuation, survival, decitabine, azacitidine \\ Received: March 17, $2017 \quad$ Accepted: April 28, $2017 \quad$ Published: May 29, 2017 \\ Copyright: Kim et al. This is an open-access article distributed under the terms of the Creative Commons Attribution License 3.0 \\ (CC BY 3.0), which permits unrestricted use, distribution, and reproduction in any medium, provided the original author and source \\ are credited.
}

\section{ABSTRACT}

It is often difficult to continue treatment with hypomethylating agent(HMA) in clinical practice because of problems such as toxicities, poor economics, etc. We compared clinical outcomes of those patients who continued HMA and those who discontinued HMA because of other causes, and evaluated factors associated with survival in those patients who discontinued HMA.

Patients were divided into two groups: treatment failure, those who stopped treatment due to disease progression; and discontinuation, those who discontinued treatment because of other causes.

The median progression free survival(PFS) was 9.2 months (range 7.7 10.7 months) vs 28.9 months (range 22.6 - 35.2) in the treatment failure and discontinuation groups, respectively $(P<0.001)$. In a multivariate analysis, a lower 


\begin{abstract}
risk by WPSS was an independent predictive factor for a longer PFS, and a lower risk by WPSS and median number of HMA cycles greater than seven were independent predictive factors for longer overall survival(OS) only in the discontinuation group. Patients who discontinued HMA without disease progression showed a prolonged survival than those who failed HMA treatment. Especially, a lower risk by WPSS and longer duration of HMA treatment may be predictive factors for a longer PFS and OS in patients who discontinued HMA.
\end{abstract}

\section{INTRODUCTION}

Myelodysplastic syndromes (MDS) are a heterogeneous collection of clonal hematopoietic malignancies that primarily affect the elderly and are characterized by bone marrow failure and dysplasia [1]. They are characterized by poor overall survival (OS) due to ineffective hematopoiesis, progressive cytopenias, and transformation to acute myeloid leukemia (AML). The hypomethylating agents (HMAs), azacitidine and decitabine, are commonly and effectively used to treat MDS; about half of high risk patients who were not eligible for allogeneic stem cell transplantation had hematologic improvement and a survival benefit with azacitidine for about 2 years [2-7]. Although treatment with HMA may prolong survival in patients with a poor prognosis, there are still a significant proportion of patients with MDS who do not respond to therapy with HMA and patients who lose response or progress on therapy [810]. According to previous studies, prolonged treatment duration may contribute to survival benefit $[4,11]$. The optimal duration of treatment is unknown, but continued therapy for as long as a response is maintained is generally recommended [12]. Continuation of therapy is particularly important in patients who achieve a complete response (CR). For those patients who stop HMA treatment, most lose any response [13].

There are known prognostic factors for survival in MDS. The Global M.D. Anderson Scoring System (MDGSS) risk model, which includes poor performance, older age, thrombocytopenia, anemia, increased bone marrow blasts, leukocytosis, chromosome 7 or complex $(\geq 3)$ abnormalities, and prior transfusions, allows risk assessment of any patient, regardless of prior therapy, at different time points in the course of the disease [14]. It was found to be independently predictive of outcome in patients with higher-risk as well as lower-risk MDS [15, 16]. Age, bone marrow blast count, and cytogenetics were found to have prognostic value in lower or higher risk patients who were treated with HMA $[17,18]$.

However, in clinical practice, though not in clinical trials, it is difficult to continue treatment of HMA because of problems such as toxicity, poor economics, comorbidities, compliance issues, etc. For those reasons, discontinuation of HMA before disease progression often happens in the clinical setting. That said, sometimes patients who stop treatment without progression show a long term survival. Cabrero et al. suggested that those patients who received more than 12 courses of HMA therapy and did not have high-risk cytogenetics had a significantly longer OS and tended to also have longer progression-free survival (PFS) [19]. On the other hand, there is a poor outcome in patients with lower-risk MDS who fail HMA treatment (median survival, 17 months) [15]. So, there are some questions about survival in MDS patients treated with HMA in clinical situations. Are there any differences of clinical outcomes between patients who continued HMA until disease progression and those who discontinued HMA because of other causes? And which factors will be helpful to predict survival in patients who discontinued HMA without disease progression? The purpose of this study was to look for answers to the above questions.

\section{RESULTS}

\section{Patient characteristics}

From January 2001 through October 2013, a total of 335 patients were collected in this study and 246 patients were analyzed. All estimated patients were diagnosed with MDS and received HMA as first line treatment. Among the excluded eighty nine patients, 46 received allogenic stem cell transplantation, 35 received less than 4 cycles of HMA, 4 did not have satisfactory raw medical records, and 4 were receiving ongoing HMA treatment. One hundred thirteen patients stopped HMA therapy because of disease progression, but 133 patients stopped HMA therapy because of other causes, such as toxicities, costs, patient refusal, planned schedule, etc. Twenty seven patients suffered from toxicities of chemotherapy, 82 refused treatment because of economics or personal reasons, and some patients were to receive 12 months of HMA chemotherapy as directed by their physicians. Additionally, 24 patients were lost to follow-up. After confirmed progression or relapse, salvage therapies were applied to patients. Chemotherapy was given to forty patients in the treatment failure group and 24 in the discontinuation group. No treatment or supportive care was administered in fifty and eighty one patients in both group, respectively. Further, information regarding salvage therapy could not be found in twenty three and twenty eight patients in the two groups, respectively. Detailed salvage therapy is presented in Figure 1.

The median age of the patients was 68 years (range 24-92 years) and the male to female ratio was 1.9:1.0. One 
hundred seventy five patients were treated with azacitidine (71.1\%) and 71 patients with decitabine (28.9\%). The median number of HMA cycles was 7 (range 4-63 cycles). All patients were stratified by IPSS, WPSS, and IPSS-R risk models.

\section{Comparison of treatment outcomes between the two groups}

The comparison of characteristics between the treatment failure and discontinuation groups is shown in Table 1 . The percentage of patients with lower than 5\% bone marrow myeloblasts was $37.9 \%$ in the treatment failure group and $62.1 \%$ in the discontinuation group $(P=$
0.015). Patients who were at lower risk by IPSS or WPSS were documented in the discontinuation group more than in the treatment failure group. The treatment failure and discontinuation groups included 60 and 87 , or 41 and 72 low risk patients, depending on the use of IPSS or WPSS, respectively $(P=0.050$ and 0.005$)$. However, patients included in the treatment failure group received salvage chemotherapy more than those in the discontinuation group $(62.5 \%$ vs $37.5 \%, P=0.006)$. The best responses were documented in all patients. $41.5 \%$ of patients were assessed with PR or more than PR, and $44.3 \%$ of patients had stable disease (SD) or only HI. The median PFS was 14.2 months (range 11.9-16.5) and the median OS was 30.7 months (range 25.3-36.1) in all patients.

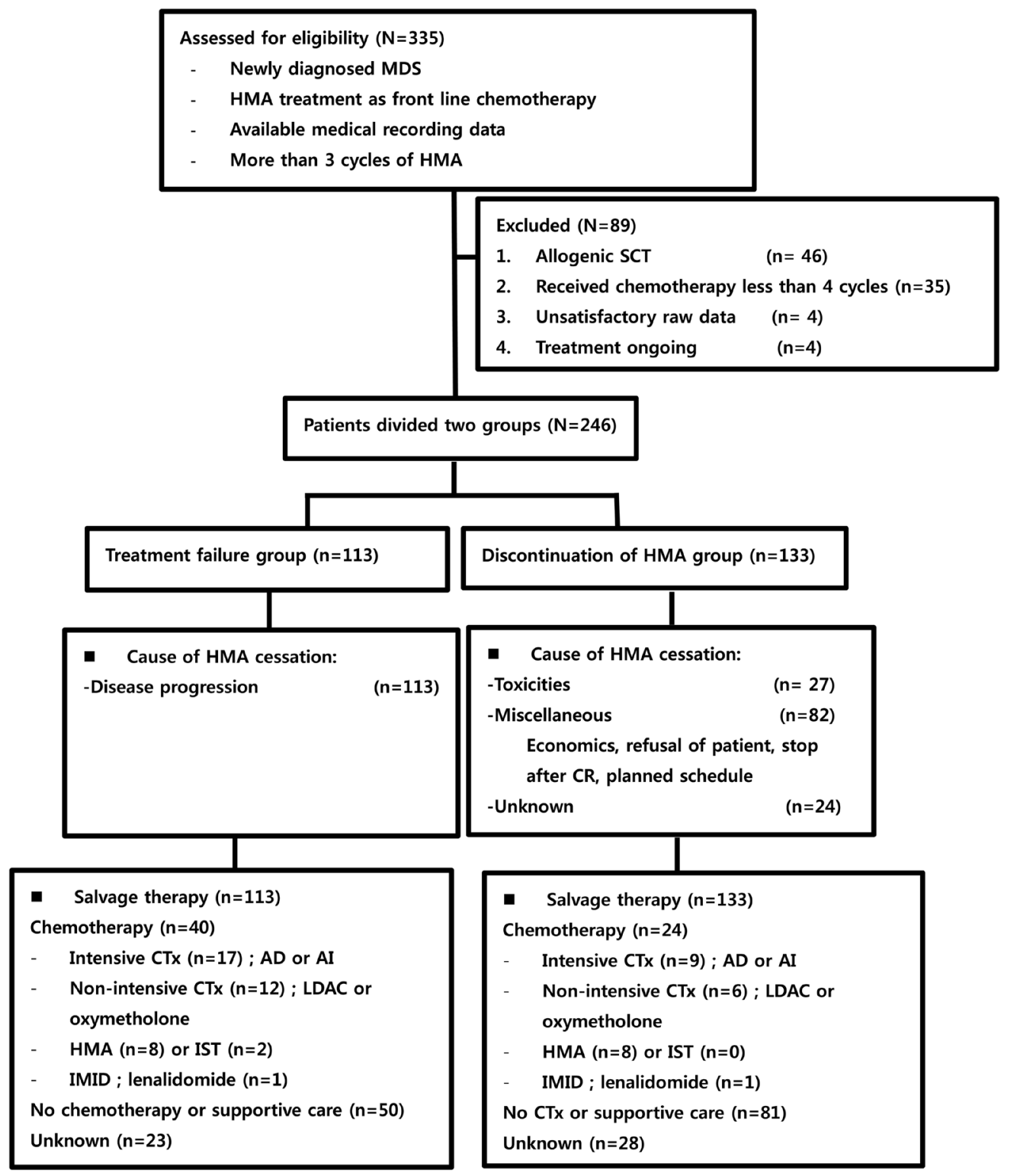

Figure 1: Study selection and flow chart. MDS: myelodysplastic syndrome; HMA: hypomethylating agents; CR: complete response; CTx: chemotherapy; AD: cytarabine plus daunorubicin; AI: cytarabine plus idarubicin; LDAC: low dose cytarabine; IST: immune suppressive therapy; IMID: immune modulatory drug. 
Table 1: Comparison of characteristics between treatment failure and discontinuation of HMA group

\begin{tabular}{|c|c|c|c|}
\hline Value $(\%)$ & Treatment failure group $(n=113)$ & Discontinuation of HMA group $(n=133)$ & $P$-value \\
\hline \multicolumn{4}{|l|}{ Age, years } \\
\hline$<65$ & $40(41.7)$ & $56(58.3)$ & \multirow[t]{2}{*}{0.283} \\
\hline$\geq 65$ & $73(48.7)$ & $77(51.3)$ & \\
\hline \multicolumn{4}{|l|}{$\operatorname{Sex}(\%)$} \\
\hline Male & $73(45.6)$ & $87(54.4)$ & \multirow[t]{2}{*}{0.894} \\
\hline Female & $40(46.5)$ & $46(53.5)$ & \\
\hline \multicolumn{4}{|l|}{ ECOG } \\
\hline$<2$ & $97(46.0)$ & $114(54.0)$ & \multirow[t]{2}{*}{0.434} \\
\hline$\geq 2$ & $10(55.6)$ & $8(4.4)$ & \\
\hline \multicolumn{4}{|l|}{ BM blast, $\%$} \\
\hline$<5$ & $44(37.9)$ & $72(62.1)$ & \multirow[t]{2}{*}{0.015} \\
\hline$\geq \mathbf{5}$ & $67(53.6)$ & $58(46.4)$ & \\
\hline \multicolumn{4}{|l|}{ Cytogenetics } \\
\hline Good & $24(393)$ & $37(607)$ & \multirow{3}{*}{0.134} \\
\hline Intermediate & $28(58.3)$ & $20(41.7)$ & \\
\hline Poor & $21(52.4)$ & $20(48.8)$ & \\
\hline \multicolumn{4}{|l|}{ Hemoglobin, g/dL } \\
\hline$<8.0$ & $54(45.0)$ & $66(55.0)$ & \multirow[t]{2}{*}{0.730} \\
\hline$\geq 8.0$ & $59(47.2)$ & $66(52.8)$ & \\
\hline \multicolumn{4}{|l|}{ Platelet, $10^{3} / \mathrm{uL}$} \\
\hline$<\mathbf{5 0}$ & $46(48.4)$ & $49(51.6)$ & \multirow[t]{2}{*}{0.566} \\
\hline$\geq \mathbf{5 0}$ & $67(44.7)$ & $83(55.3)$ & \\
\hline \multicolumn{4}{|l|}{ IPSS risk, n (\%) } \\
\hline Lower risk & $60(40.8)$ & $87(59.2)$ & \multirow[t]{2}{*}{0.050} \\
\hline Higher risk & $53(53.5)$ & $46(46.5)$ & \\
\hline \multicolumn{4}{|l|}{ WPSS risk, n (\%) } \\
\hline Lower risk & $41(36.3)$ & $72(63.7)$ & \multirow[t]{2}{*}{0.005} \\
\hline Higher risk & $72(54.1)$ & $61(45.9)$ & \\
\hline \multicolumn{4}{|l|}{ IPSS-R risk, n (\%) } \\
\hline Lower risk & $35(44.3)$ & $44(42.6)$ & \multirow[t]{2}{*}{0.121} \\
\hline Higher risk & $34(57.6)$ & $25(42.4)$ & \\
\hline \multicolumn{4}{|l|}{ HMA treatment } \\
\hline Azacitidine & $81(46.3)$ & $94(53.7)$ & \multirow[t]{2}{*}{0.862} \\
\hline Decitabine & $32(45.1)$ & $39(54.9)$ & \\
\hline \multicolumn{4}{|l|}{ Number of HMA cycles } \\
\hline$<7$ cycles & $48(40.7)$ & $70(59.3)$ & \multirow[t]{2}{*}{0.112} \\
\hline$\geq 7$ cycles & $65(50.8)$ & $63(49.2)$ & \\
\hline \multicolumn{4}{|l|}{ Response } \\
\hline$<\mathbf{P R}$ & $53(43.8)$ & $68(56.2)$ & 0.527 \\
\hline$\geq \mathbf{P R}$ & $49(48.0)$ & $53(52.0)$ & \\
\hline Salvage therapy & & & \\
\hline Chemotherapy & $40(62.5)$ & $24(37.5)$ & 0.006 \\
\hline No or supportive care & $50(38.2)$ & $81(61.8)$ & \\
\hline Unknown & $23(45.1)$ & $28(54.9)$ & \\
\hline
\end{tabular}

HMA: hypomethylating agents, ECOG: Eastern Cooperative Oncology Group performance status, BM: bone marrow, IPSS: international prognostic scoring system, WPSS: WHO adapted prognostic scoring system, IPSS-R: revised international prognostic scoring system, PR: partial response. 
Table 2: Univariate and multivariate analysis for survival in all patients $(\mathrm{n}=246)$

\begin{tabular}{|c|c|c|c|c|c|c|c|c|c|c|}
\hline \multirow{3}{*}{ Value } & \multicolumn{4}{|c|}{ Univariate } & \multicolumn{6}{|c|}{ Multivariate } \\
\hline & \multicolumn{2}{|c|}{ 3yrs PFS } & \multicolumn{2}{|c|}{ 3yrs OS } & \multicolumn{3}{|c|}{ 3yrs PFS } & \multicolumn{3}{|c|}{ 3yrs OS } \\
\hline & & $P$-value & & $P$-value & HR & 95\% C.I & $P$-value & HR & 95\% C.I & $P$-value \\
\hline \multicolumn{11}{|l|}{ Age, years } \\
\hline$<65$ & 28.7 & 0.463 & 51.6 & 0.016 & & & & & & \\
\hline$\geq 65$ & 24.2 & & 36.0 & & & & & 0.579 & $0.343 \sim 0.978$ & 0.041 \\
\hline \multicolumn{11}{|l|}{ BM blast, \% } \\
\hline$<5$ & 37.8 & $<0.001$ & 60.1 & $<0.001$ & & & & & & \\
\hline$\geq 5$ & 11.4 & & 24.2 & & 0.826 & $0.428 \sim 1.594$ & 0.568 & 0.475 & $0.214 \sim 1.053$ & 0.067 \\
\hline \multicolumn{11}{|l|}{ Cytogenetics } \\
\hline Good & 43.8 & $<0.001$ & 49.6 & $<0.001$ & & & & & & \\
\hline Intermediate & 9.1 & & 28.5 & & 0.617 & $0.328 \sim 1.161$ & 0.043 & 0.304 & $0.146 \sim 0.634$ & 0.001 \\
\hline Poor & 11.1 & & 17.3 & & 1.268 & $0.694 \sim 2.318$ & 0.134 & 0.176 & $0.080 \sim 0.388$ & $<0.001$ \\
\hline \multicolumn{11}{|l|}{ Platelet, $10^{3} / \mathrm{uL}$} \\
\hline$<\mathbf{5 0}$ & 21.1 & 0.397 & 34.1 & 0.051 & & & & & & \\
\hline$\geq \mathbf{5 0}$ & 29.1 & & 48.0 & & & & & 1.376 & $0.809 \sim 2.342$ & 0.239 \\
\hline \multicolumn{11}{|l|}{ IPSS risk, n (\%) } \\
\hline Lower risk & 34.3 & $<0.001$ & 57.3 & $<0.001$ & & & & & & \\
\hline Higher risk & 12.6 & & 19.6 & & 1.298 & $0.697 \sim 2.417$ & 0.411 & 1.619 & $0.863 \sim 3.037$ & 0.133 \\
\hline \multicolumn{11}{|l|}{ WPSS risk, n (\%) } \\
\hline Lower risk & 44.7 & $<0.001$ & 65.3 & $<0.001$ & & & & & & \\
\hline Higher risk & 9.2 & & 22.2 & & 0.509 & $0.245 \sim 1.058$ & 0.070 & 0.290 & $0.112 \sim 0.752$ & 0.011 \\
\hline \multicolumn{11}{|l|}{ IPSS-R risk, n (\%) } \\
\hline Lower risk & 36.2 & $<0.001$ & 49.3 & $<0.001$ & & & & & & \\
\hline Higher risk & 4.6 & & 8.5 & & 0.637 & $0.369 \sim 1.099$ & 0.105 & 1.425 & $0.722 \sim 2.812$ & 0.308 \\
\hline \multicolumn{11}{|l|}{ HMA treatment } \\
\hline$<7$ cycles & 23.3 & 0.014 & 34.7 & 0.002 & & & & & & \\
\hline$\geq 7$ cycles & 28.2 & & 49.1 & & 0.860 & $0.560 \sim 1.320$ & 0.491 & 2.301 & $1.401 \sim 3.778$ & 0.001 \\
\hline \multicolumn{11}{|l|}{ HMA group } \\
\hline Treatment failure & 6.8 & $<0.001$ & 27.8 & $<0.001$ & & & & & & \\
\hline Discontinuation & 44.0 & & 54.8 & & 5.864 & 3.589 9.579 & $<0.001$ & 2.212 & $1.255 \sim 3.897$ & 0.006 \\
\hline
\end{tabular}

3 yrs PFS: 3 years progression free survival rates; 3 yrs OS: 3 years overall survival rates; HR: hazard ratio; BM: bone marrow; IPSS: international prognostic scoring system; WPSS: WHO adapted prognostic scoring system; IPSS-R: revised international prognostic scoring system; HMA: hypomethylating agents.

The differences in survival between lower and higher risk MDS are as follows. In lower risk MDS, 3 years PFS of treatment failure group and discontinuation group are $6.1 \%$ and $54.5 \%$, respectively $(P<0.001)$ and 3 years OS of treatment failure group and discontinuation group are $36.2 \%$ and $72.6 \%,(P<0.001)$ respectively. In higher risk MDS, 3 years PFS of treatment failure group and discontinuation group are $7.8 \%$ and $19.5 \%$, respectively $(p=0.001)$ and 3 years OS of treatment failure group and discontinuation group are $19.3 \%$ and $20.1 \%,(p=0.994)$ respectively.

In a univariate analysis, bone marrow myeloblasts less than 5\%, good cytogenetics, lower risk by IPSS, WPSS, and IPSS-R, median number of HMA cycles greater than 7, and discontinuation of HMA were significant predictors of a longer PFS (Table 2). An age less than 65 years, median number of HMA cycles more than 7 , bone marrow myeloblasts less than $5 \%$, good 
cytogenetics, lower risk by IPSS, WPSS, and IPSS-R, and discontinuation of HMA were significant predictors of longer OS in all patients as well (Table 2). In a multivariate analysis, intermediate cytogenetics was an independent poor risk factor for PFS (Hazard ratio (HR): $0.617, P=0.043$ ) and discontinuation of HMA was an independent good risk factor for PFS (HR: 5.864, $P<$ $0.001)$. An age greater than 65 years, poor cytogenetics, a higher risk by WPSS (HR: 0.579, $P=0.041$, HR: 0.176, $P<0.001$, and HR: $0.290, P=0.011$, Table 2), median number of HMA cycles more than 7 , and discontinuation of HMA were also independent good risk factors for OS (HR: 2.301, $P<0.001$, and HR: 2.212, $P=0.006$, Table 2 ). Of note, the median PFS was 9.2 months (range $7.7-$ 10.7 months) vs 28.9 months (range 22.6 - 35.2) in the treatment failure and discontinuation groups, respectively $(P<0.001$, Figure 2A). The median OS was 22.1 months (range 18.2 - 26.0 months) vs 50.1 months (range $23.9-$ 76.3 months $)$ in the two groups, respectively $(P<0.001$, Figure 2B).

\section{Factors associated with survival in the discontinuation group}

However, further analyses proceeded to estimate predictive factors for a longer PFS and OS in the discontinuation group $(n=133)$. In a univariate analysis, some factors, such as bone marrow myeloblasts $<5 \%$, good cytogenetics, lower risk by IPSS, WPSS, and IPSS-R, and median number of HMA cycles more than 7 , were significant predictive factors for a longer PFS and OS in the discontinuation group (Table 3 ). In a multivariate analysis, a higher risk by WPSS was an independent poor risk factor for PFS (HR: $0.244, P=0.026$ ) and OS (HR: $0.146, P=0.014)$. A median number of HMA cycles more than 7 was an independent good risk factor for OS (HR: $4.043, P=0.005$ ) in only the discontinuation group (Table 3).

\section{DISCUSSION}

In this retrospective analysis, patients who discontinued HMA without disease progression showed a superior PFS and OS than those who failed HMA treatment. Comparing the two groups, there were more patients with bone marrow myeloblasts $<5 \%$ and a lower risk by IPSS and WPSS in the discontinuation group than in the treatment failure group (Table 1). These differences in baseline characteristics might affect PFS and OS in both groups. So, further evaluation proceeded to confirm whether discontinuation of HMA treatment affects differences of survival between the two groups or not. In a multivariate analysis, independent prognostic factors for OS were age, bone marrow myeloblast percent, cytogenetic risk, number of HMA cycles, and a prognostic risk model, which have already been documented as factors in previous studies [14-16]. Especially, discontinuation or failure of HMA treatment was found as an independent prognostic factor for PFS and $\mathrm{OS}$ in a multivariate analysis. However, more patients with lower risk MDS were included in the discontinuation group than the treatment failure group, which was likely due to physician discretion. Until now, some previous studies reported that long term use of HMA improved survival in patients with higher risk MDS [21, 22]. The recommendations were to continue azacitidine until progression in patients achieving at least stable disease with hematologic improvement [23]. But, there are no data supporting the interruption of azacitidine before progression in responding patients. So, in real clinical practice, physicians tend to continue HMA treatment in higher risk MDS; but, patients with lower risk MDS often stopped more frequently, especially as a result of other causes such as toxicities, poor economics, comorbidities, compliance, etc., without disease progression. Actually, patients with lower risk MDS were more prominent in the discontinuation group than in the treatment failure group in our study (Table 1).

However, Cabrero $\mathrm{M}$ et al. presented a series of 16 patients with higher-risk MDS $(n=5 ; 31 \%)$ or AML $(n=11 ; 69 \%)$ who achieved PR $(n=1)$ or CR $(n=$ $15)$ and stopped HMA therapy [19]. They suggested that patients who received 12 cycles of therapy or more had a significantly better OS (median: 20 months [95\% CI: 12-27]) than those who received fewer than 12 cycles (median: 4 months [95\% CI: $1-8])(P=0.043)$. Poorrisk cytogenetics were also associated with a lower 1 -year OS (33\% versus 69\%; $P=0.046)$. Jabbour EJ et al, reported about outcomes of HMA failure. After a median follow-up of 21 months from decitabine failure, $13(15 \%)$ patients remained alive; the median survival was 4.3 months [16]. And from a report on behalf of the MDS Clinical Research Consortium, the median transformation-free survival and OS after HMA failure were 15 months and 17 months, respectively. The estimated 12-month survival rates were 90\%, $77 \%, 37 \%$ and $39 \%$, respectively, for patients with low-risk, intermediate-1-risk, intermediate-2-risk, and high-risk disease by the IPSS. Baseline neutropenia, intermediate-risk and poor-risk baseline karyotype, and lack of response to HMA were found to be associated with a higher risk of disease progression [15]. In this study, lower risk by WPSS was an independent risk factor for a longer PFS, and lower risk by WPSS and a median number of HMA cycles greater than 7 were independent risk factors for a longer OS only in the discontinuation group (Table 3). According to our results, discontinuation of HMA can be considered carefully in patients who were shown as lower risk by prognostic models and who sustained a good response with long term HMA. However, there were some limitations to this study. As our data were collected 
a

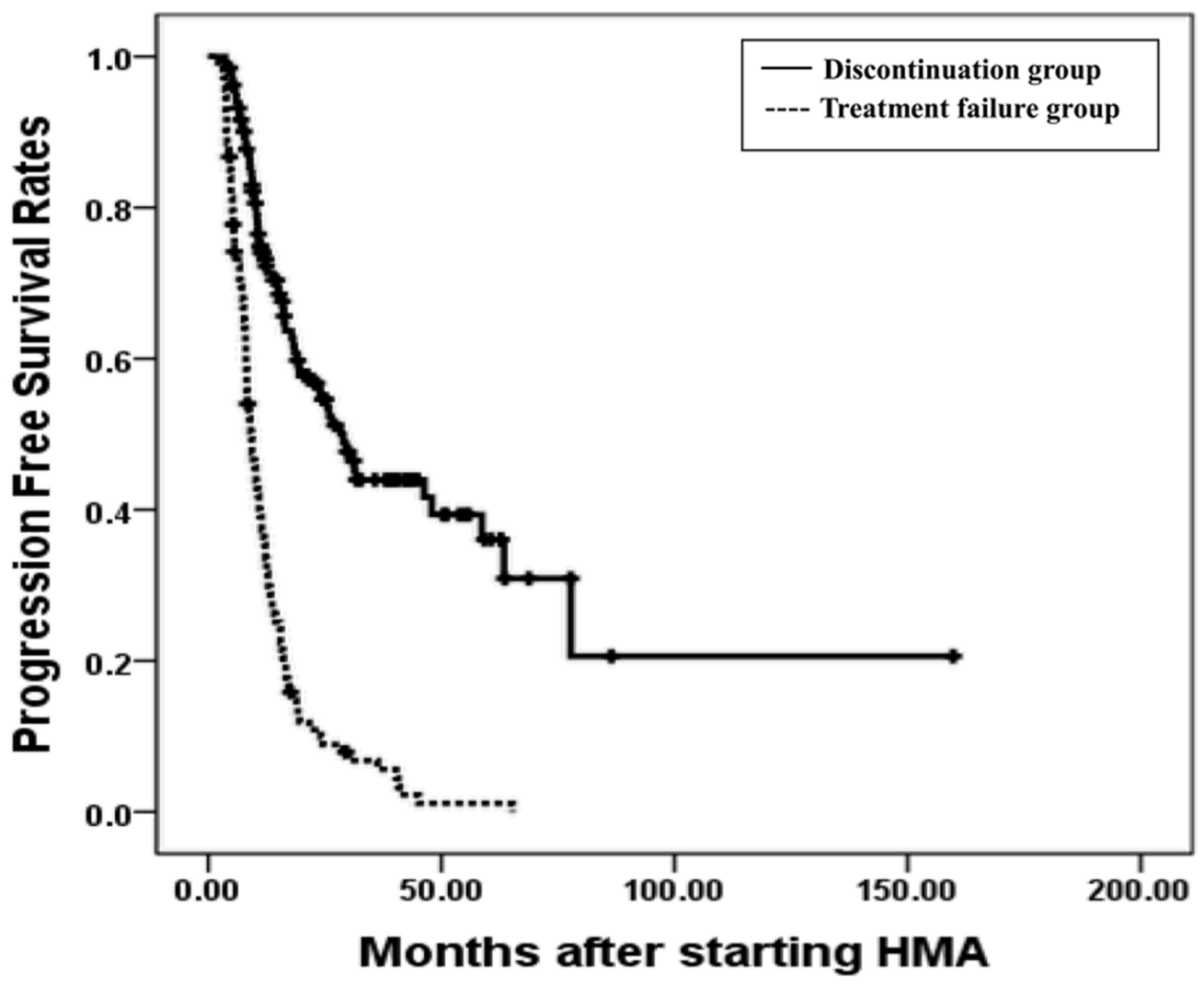

$\mathrm{b}$

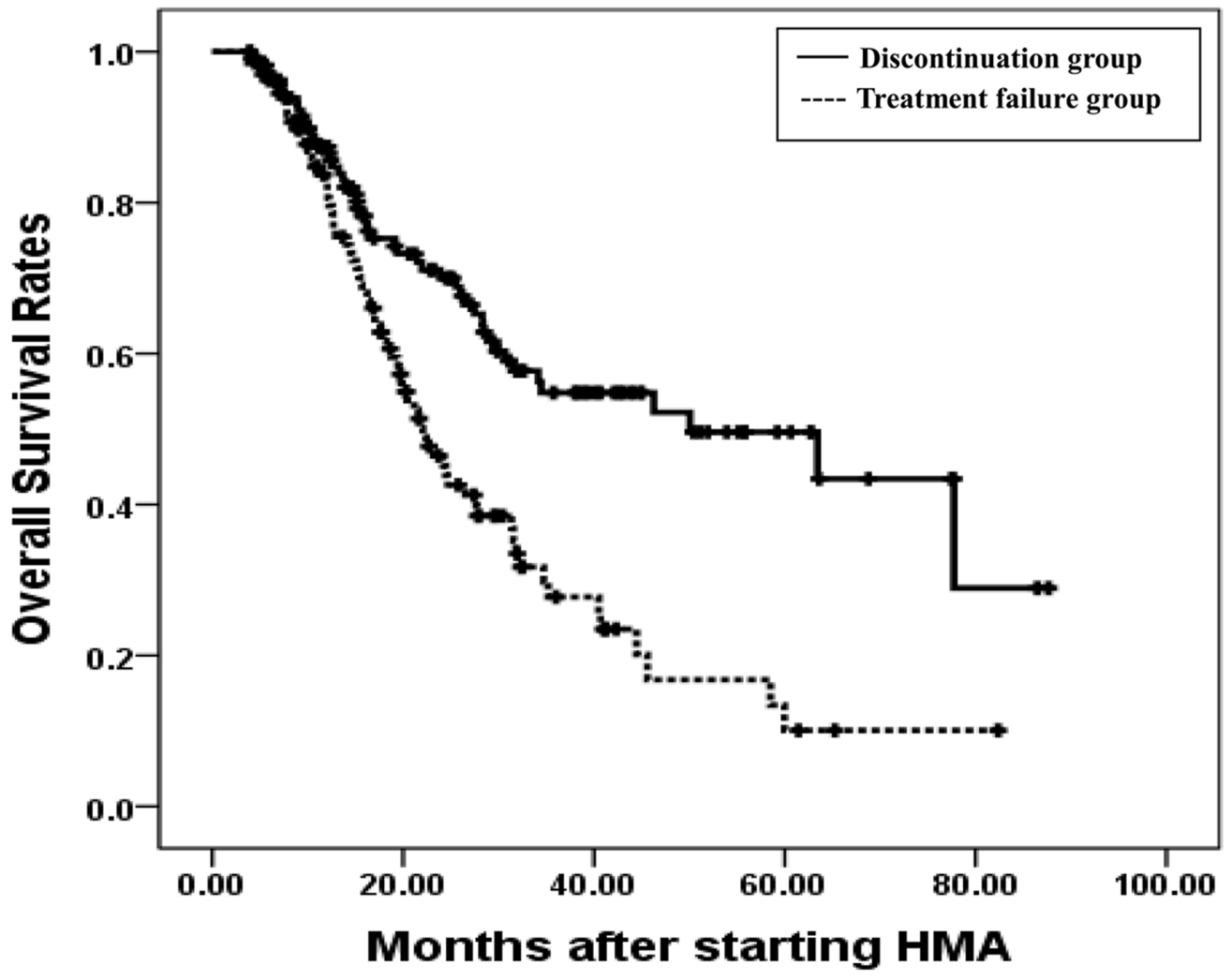

Figure 2: The 3 years progression free survival rates (PFS) and overall survival rates(OS). The 3 years progression free survival rates (PFS) in treatment failure and discontinuation group were $6.8 \%$ and $44.0 \%, P<0.001$, respectively (a), and the 3 years overall survival rates (OS) in two groups were $27.8 \%$ and $54.8 \%, P<0.001$, respectively (b). 
Table 3: Univariate and multivariate analyses for survival in discontinuation group (n=133)

\begin{tabular}{|c|c|c|c|c|c|c|c|c|c|c|}
\hline \multirow{3}{*}{ Value } & \multicolumn{4}{|c|}{ Univariate } & \multicolumn{6}{|c|}{ Multivariate } \\
\hline & \multicolumn{2}{|c|}{ 3yrs PFS } & \multicolumn{2}{|c|}{ 3yrs OS } & \multicolumn{3}{|c|}{ 3yrs PFS } & \multicolumn{3}{|c|}{ 3yrs OS } \\
\hline & & $P$-value & & $P$-value & HR & 95\% C.I & $P$-value & HR & 95\% C.I & $P$-value \\
\hline \multicolumn{11}{|l|}{ BM blast, \% } \\
\hline$<5$ & 56.8 & 0.002 & 69.5 & $<0.001$ & & & & & & \\
\hline$\geq 5$ & 20.9 & & 30.8 & & 1.762 & $0.569 \sim 5.458$ & 0.326 & 1.098 & $0.321 \sim 3.756$ & 0.882 \\
\hline \multicolumn{11}{|l|}{ Cytogenetics } \\
\hline Good & 69.2 & 0.001 & 67.3 & 0.044 & & & & & & \\
\hline Intermediate & 26.5 & & 38.9 & & 0.594 & $0.146 \sim 2.419$ & 0.467 & 0.488 & $0.097 \sim 2.458$ & 0.385 \\
\hline Poor & 17.7 & & 34.0 & & 1.038 & $0.276 \sim 3.898$ & 0.956 & 0.574 & $0.089 \sim 3.708$ & 0.560 \\
\hline \multicolumn{11}{|l|}{ IPSS risk, n (\%) } \\
\hline Lower risk & 54.5 & 0.001 & 70.5 & $<0.001$ & & & & & & \\
\hline Higher risk & 19.5 & & 20.1 & & 1.124 & $0.407 \sim 3.105$ & 0.822 & 0.782 & $0.276 \sim 2.212$ & 0.643 \\
\hline \multicolumn{11}{|c|}{ WPSS risk, n (\%) } \\
\hline Lower risk & 65.4 & $<0.001$ & 76.0 & $<0.001$ & & & & & & \\
\hline Higher risk & 15.5 & & 26.4 & & 0.244 & $0.071 \sim 0.842$ & 0.026 & 0.146 & $0.032 \sim 0.679$ & 0.014 \\
\hline \multicolumn{11}{|c|}{ IPSS-R risk, n (\%) } \\
\hline Lower risk & 59.3 & $<0.001$ & 61.9 & 0.032 & & & & & & \\
\hline Higher risk & 8.7 & & 18.5 & & 0.405 & $0.104 \sim 1.570$ & 0.191 & 1.387 & $0.232 \sim 8.283$ & 0.720 \\
\hline \multicolumn{11}{|l|}{ HMA treatment } \\
\hline$<7$ cycles & 37.3 & 0.044 & 46.1 & 0.007 & & & & & & \\
\hline$\geq 7$ cycles & 51.0 & & 64.7 & & 0.967 & $0.436 \sim 2.142$ & 0.934 & 4.043 & $1.525 \sim 10.720$ & 0.005 \\
\hline
\end{tabular}

3 yrs PFS: 3 years progression free survival rates; 3 yrs OS: 3 years overall survival rates; HR: hazard ratio; BM: bone marrow; IPSS: international prognostic scoring system; WPSS: WHO adapted prognostic scoring system; IPSS-R: revised international prognostic scoring system; HMA: hypomethylating agents.

retrospectively, analyzed raw data were often not sufficient or fully exact. The forty four and sixty four patients categorized by IPSS and WPSS, respectively, could not be analyzed for survival by IPSS-R because of insufficient cytogenetic data. Additionally, twenty two patients had insufficient medical data because of follow up loss.

In conclusion, patients without disease progression who discontinued HMA showed a prolonged survival compared to those who failed HMA treatment in clinical practice. In particular, patients with a lower risk by WPSS and long term use of HMA for at least seven cycles may have a prolonged PFS and OS by discontinuing HMA. However, further studies are needed to determine which patients can be considered for discontinuation of HMA and how many cycles of HMA treatment may be optimal for longer survival in true clinical practice.

\section{MATERIALS AND METHODS}

\section{Patients and study design}

A total of 335 patients who satisfied the following criteria were enrolled at fourteen university hospitals in South Korea between January 2001 and October 2013. The medical records of this study were collected retrospectively from a nationwide registry, which was performed with the AML/MDS working party in South Korea. All included patients had been newly diagnosed with MDS and were treated with HMAs, such as azacitidine or decitabine, as front line therapy continuously for at least 4 cycles. Patients were excluded from this study if they had received allogenic stem cell transplantation, showed early death, or ceased treatment without a response evaluation before at least 4 cycles of 
HMA. Patients with unsatisfactory raw medical recording data were also excluded. The study flow chart is shown in Figure 1. All patients were treated with decitabine $20 \mathrm{mg} /$ $\mathrm{m}^{2}$ for 5 days every 4 weeks or azacitidine $75 \mathrm{mg} / \mathrm{m}^{2}$ for 7 days every 4 weeks.

Patients were divided into two groups: treatment failure, which are those who discontinued HMA treatment due to disease progression, and the discontinuation group, those who stopped HMA treatment because of other causes such as toxicity, poor economics, comorbidities, compliance, etc. The toxicity was determined by the investigator's judgment at each institution when further treatment is impossible because if hematologic or non-hematologic toxicity is at least National Cancer Institute (NCI) Common Terminology Criteria for Adverse Events (CTCAE) grade 3 to 4 . All patients were allowed salvage therapy at the discretion of a clinician. Cytogenetic analysis from bone marrow samples of patients was carried out according to standard procedures. Where possible, 20 or more metaphases from each patient were analyzed in order to demonstrate the clonal nature of the aberrations. The cytogenetic risk, which was determined by conventional cytogenetics, was categorized as good, intermediate, or high risk. Good risk included normal cytogenetics and diploid/loss of chromosome $\mathrm{Y}$, deletion in $5 \mathrm{q}$ only, and deletion in $20 \mathrm{q}$ only. High risk factors included complex abnormalities that involved $\geq 3$ changes or a chromosome 7 abnormality, either alone or with other abnormalities. Intermediate risk was defined as not meet the conditions of either low or high risk. Treatment response was assessed with the 2006 modified international working group response criteria. Besides induction of $\mathrm{CR}$ and partial response (PR), achievement of hematologic improvement (HI) according to the International Working Group (IWG) 2006 criteria, i.e., improvement in cytopenias (mainly anemia and/or thrombocytopenia), should be considered indicative of response to treatment because it has been shown to be associated with a prolongation of survival [20]. Responses to HMA and subsequent therapies were coded according to the 2006 IWG Criteria for response assessment in patients with MDS. Response evaluation was performed at least every 4 cycles. BM assessment was performed for patients with BM blasts greater than 5\% after 3-4 cycles of treatment. Disease progression was defined as at least a $50 \%$ decrease from the maximum response in granulocytes or platelets, a reduction in hemoglobin by more than $2 \mathrm{~g} / \mathrm{dL}$, or change to transfusion-dependence. Treatment failure was defined as no response after at least 4 cycles of therapy, loss of response, progression to higher-risk MDS categories, or transformation to acute myeloid leukemia (AML).

\section{Statistical analysis}

PFS was defined as the duration from the start date of HMA therapy to the date of disease progression, relapse, or death from any causes. OS was defined as the duration from the start date of HMA therapy to the date of death from any cause or the final follow-up date. Survival probabilities were calculated using the KaplanMeier method, assessed from starting HMA therapy, and compared using the log-rank test. Univariate and multivariate analyses were performed to identify potential prognostic factors associated with PFS and OS. In this study, previous reported prognostic factors such as poor performance, older age, thrombocytopenia, anemia, increased bone marrow blasts, leukocytosis, chromosome 7 or complex ( $\geq 3$ ) abnormalities, prior transfusions, and risk models, including the international prognostic scoring system (IPSS), WHO adapted prognostic scoring system (WPSS), and revised IPSS (IPSS-R) risk model, were analyzed; treatment failure, discontinuation of HMA, and number of HMA cycles were additionally estimated in this study. The Cox proportional hazard regression analysis was used for PFS and OS. Information about baseline medical status and treatment modalities were collected from the medical records. Approval for these studies was obtained from the Institutional Review Board.

\section{Abbreviations}

HMA: hypomethylating agent; PFS: progression free survival; OS: overall survival; MDS: myelodysplastic syndrome; AML: acute myeloid leukemia; CR: complete response; PR: partial response; HI: hematologic improvement; IWG: International Working group; IPSS: international prognostic scoring system; WPSS: WHO adapted prognostic scoring system; IPSS-R: revised IPSS; SD: stable disease; HR: hazard ratio.

\section{Author contributions}

DJ and HS analyzed the clinical data and wrote the manuscript. HS designed the study. DJ contributed to conception of the study and analyzed the clinical data. JH, SK, HJ, JW, DY, H, HS, SM, WS, Y, MH, JH, SH, MK contributed patients, provided substantial contributions to the acquisition and interpretation of data. DJ and HS was involved in revising the manuscript critically for intellectual content and gave final approval for the submission of the manuscript. All authors reviewed the final manuscript.

\section{ACKNOWLEDGMENTS}

The authors would like to thank 'The Korean Society of Hematology AML/MDS working party’ for 
contributing patients to the study and data managers for their great work.

\section{CONFLICTS OF INTEREST}

The authors have no conflicts of interest.

\section{FUNDING}

Not applicable.

\section{REFERENCES}

1. Vardiman JW, Thiele J, Arber DA, Brunning RD, Borowitz MJ, Porwit A, Harris NL, Le Beau MM, HellströmLindberg E, Tefferi A, Bloomfield CD. The 2008 revision of the World Health Organization (WHO) classification of myeloid neoplasms and acute leukemia: rationale and important changes. Blood. 2009; 114:937-951.

2. Silverman LR, Demakos EP, Peterson BL, Kornblith AB, Holland JC, Odchimar-Reissig R, Stone RM, Nelson D, Powell BL, DeCastro CM, Ellerton J, Larson RA, Schiffer $\mathrm{CA}$, et al. Randomized controlled trial of azacitidine in patients with the myelodysplastic syndrome: a study of the cancer and leukemia group B. J Clin Oncol. 2002; 20:2429-2440.

3. Steensma DP, Baer MR, Slack JL, Buckstein R, Godley LA, Garcia-Manero G, Albitar M, Larsen JS, Arora S, Cullen MT, Kantarjian H. Multicenter study of decitabine administered daily for 5 days every 4 weeks to adults with myelodysplastic syndromes: the alternative dosing for outpatient treatment (ADOPT) trial. J Clin Oncol. 2009; 27:3842-3848.

4. Fenaux P, Mufti GJ, Hellstrom-Lindberg E, Santini V, Finelli C, Giagounidis A, Schoch R, Gattermann N, Sanz G, List A, Gore SD, Seymour JF, Bennett JM, et al. Efficacy of azacitidine compared with that of conventional care regimens in the treatment of higher-risk myelodysplastic syndromes: a randomised, open-label, phase III study. Lancet Oncol. 2009; 10:223-232.

5. Lübbert M, Suciu S, Baila L, Rüter BH, Platzbecker U, Giagounidis A, Selleslag D, Labar B, Germing U, Salih HR, Beeldens F, Muus P, Pflüger KH, et al. Low-dose decitabine versus best supportive care in elderly patients with intermediate- or high-risk myelodysplastic syndrome (MDS) ineligible for intensive chemotherapy: final results of the randomized phase III study of the European Organisation for Research and Treatment of Cancer Leukemia Group and the German MDS Study Group. J Clin Oncol. 2011; 29:1987-1996.

6. Santini V. Novel therapeutic strategies: hypomethylating agents and beyond. Hematology Am Soc Hematol Educ Program. 2012; 2012:65-73.

7. Lee YG, Kim I, Yoon SS, Park S, Cheong JW, Min YH, Lee JO, Bang SM, Yi HG, Kim CS, Park Y, Kim BS, Mun
YC, et al. Comparative analysis between azacitidine and decitabine for the treatment of myelodysplastic syndromes. Br J Haematol. 2013; 161:339-347.

8. Kadia TM, Jabbour E, Kantarjian H. Failure of hypomethylating agent-based therapy in myelodysplastic syndromes. Semin Oncol. 2011; 38:682-692.

9. Ritchie EK. Safety and efficacy of azacitidine in the treatment of elderly patients with myelodysplastic syndrome. Clin Interv Aging. 2012; 7:165-173.

10. Wang R, Gross CP, Frick K, Xu X, Long J, Raza A, Galili N, Zikria J, Guan Y, Ma X. The impact of hypomethylating agents on the cost of care and survival of elderly patients with myelodysplastic syndromes. Leuk Res. 2012; 36:1370-1375.

11. Silverman LR, McKenzie DR, Peterson BL, Holland JF, Backstrom JT, Beach CL, Larson RA; Cancer and Leukemia Group B. Further analysis of trials with azacitidine in patients with myelodysplastic syndrome: studies 8421, 8921 , and 9221 by the cancer and Leukemia Group B. J Clin Oncol. 2006; 24:3895-3903.

12. Killick SB, Carter C, Culligan D, Dalley C, Das-Gupta E, Drummond M, Enright H, Jones GL, Kell J, Mills J, Mufti G, Parker J, Raj K, et al. Guidelines for the diagnosis and management of adult myelodysplastic syndromes. Br J Haematol. 2014; 164:503-525.

13. Garcia-Manero G, Fenaux P. Hypomethylating agents and other novel strategies in myelodysplastic syndromes. J Clin Oncol. 2011; 29:516-523.

14. Kantarjian H, O'Brien S, Ravandi F, Cortes J, Shan J, Bennett JM, List A, Fenaux P, Sanz G, Issa JP, Freireich EJ, Garcia-Manero G. Proposal for a new risk model in myelodysplastic syndrome that accounts for events not considered in the original International Prognostic Scoring System. Cancer. 2008; 113:1351-1361.

15. Jabbour EJ, Garcia-Manero G, Strati P, Mishra A, Al Ali NH, Padron E, Lancet J, Kadia T, Daver N, O'Brien S, Steensma DP, Sekeres MA, Gore SD, et al. Outcome of patients with low-risk and intermediate-1-risk myelodysplastic syndrome after hypomethylating agent failure: a report on behalf of the MDS Clinical Research Consortium. Cancer. 2015; 121:876-882.

16. Jabbour E, Garcia-Manero G, Batty N, Shan J, O'Brien S, Cortes J, Ravandi F, Issa JP, Kantarjian H. Outcome of patients with myelodysplastic syndrome after failure of decitabine therapy. Cancer. 2010; 116:3830-3834.

17. Prébet $\mathrm{T}$, Gore SD, Esterni B, Gardin C, Itzykson R, Thepot S, Dreyfus F, Rauzy OB, Recher C, Adès L, Quesnel B, Beach CL, Fenaux P, et al. Outcome of highrisk myelodysplastic syndrome after azacitidine treatment failure. J Clin Oncol. 2011; 29:3322-3327.

18. Prébet T, Thepot S, Gore SD, Dreyfus F, Fenaux P, Vey N. Outcome of patients with low-risk myelodysplasia after azacitidine treatment failure. Haematologica. 2013; 98:e18-e19. 
19. Cabrero M, Jabbour E, Ravandi F, Bohannan Z, Pierce S, Kantarjian HM, Garcia-Manero G. Discontinuation of hypomethylating agent therapy in patients with myelodysplastic syndromes or acute myelogenous leukemia in complete remission or partial response: retrospective analysis of survival after long-term follow-up. Leuk Res. 2015; 39:520-524.

20. Cheson BD, Greenberg PL, Bennett JM, Lowenberg B, Wijermans PW, Nimer SD, Pinto A, Beran M, de Witte TM, Stone RM, Mittelman M, Sanz GF, Gore SD, et al. Clinical application and proposal for modification of the International Working Group (IWG) response criteria in myelodysplasia. Blood. 2006; 108:419-425.

21. Silverman LR, Fenaux P, Mufti GJ, Santini V, HellströmLindberg E, Gattermann N, Sanz G, List AF, Gore SD,
Seymour JF. Continued azacitidine therapy beyond time of first response improves quality of response in patients with higher-risk myelodysplastic syndromes. Cancer. 2011; 117:2697-2702.

22. Itzykson R, Thépot S, Quesnel B, Dreyfus F, Beyne-Rauzy O, Turlure P, Vey N, Recher C, Dartigeas C, Legros L, Delaunay J, Salanoubat C, Visanica S, et al. Prognostic factors for response and overall survival in 282 patients with higher-risk myelodysplastic syndromes treated with azacitidine. Blood. 2011; 117:403-411.

23. Itzykson R, Fenaux P. Optimizing hypomethylating agents in myelodysplastic syndromes. Curr Opin Hematol. 2012; 19:65-70. 